\title{
Successful Management of Internal Carotid Artery Transection Secondary to a Gunshot Wound and Subsequent Malignant MCA Syndrome
}

\author{
George Cairns Alex Belshaw \\ Stroke Medicine, Newham University Hospital, Barts Health NHS Trust, London, UK
}

\section{Keywords}

Internal carotid artery ligation · Malignant middle cerebral artery syndrome $\cdot$ Ischaemic

stroke $\cdot$ Decompressive craniectomy

\begin{abstract}
There are over 100,000 strokes each year in the UK. A very small proportion of these can be attributed to gunshot wounds and subsequent surgical intervention. We present a rare case of a 24-year-old male patient admitted to the Emergency Department having sustained a gunshot wound to the left side of his neck. Initial imaging and surgical exploration revealed significant left-sided vertebral artery damage and a complete transection of the internal carotid artery. Following damage control surgery (DCS), the patient was admitted to ITU but had an acute neurological deterioration and was found to have suffered malignant middle cerebral artery (MCA) syndrome, requiring an urgent decompressive craniectomy. The patient's National Institutes of Health Stroke Scale (NIHSS) at this stage was 26. After a prolonged ITU stay and repatriation to a local stroke unit for intensive therapies input, the patient walked out of the hospital independently on day 106, with an improved NIHSS of 3. This case report aims to highlight the rarity of an ischaemic stroke, secondary to the DCS required for a near fatal gunshot wound, along with the importance of timely recognition of an acute deterioration following artery ligation. Additionally, it aims to examine the lifesaving surgical management of malignant MCA syndrome and in turn the significance of the shared decision-making process between clinicians, the patient, and family members, due to the high rate of poor functional outcomes following this major surgery.
\end{abstract}

\section{Karger" ${ }^{\prime \prime}$}


Cairns and Belshaw: Management of ICA Transection and Subsequent Malignant MCA Syndrome

\section{Introduction}

Over 100,000 strokes occur each year in the UK [1]. The likelihood of a stroke occurring secondary to trauma is as low as $2.5 \%$; the largest cause of which remains road traffic accidents - attributable to approximately $50 \%$ of overall cases. Only $7 \%$ of strokes secondary to trauma are caused by gunshot wounds (GSWs); thus remaining rare events in the UK [2].

Penetrating trauma to the neck may cause carotid injuries, which carry a mortality rate of close to $100 \%$ when left untreated [3]. Carotid artery injuries are usually managed with either vessel repair or ligation, the latter of which is used more commonly when patients are haemodynamically unstable or access to the artery is more difficult [4]. Artery ligation carries a greater risk of subsequent stroke, with patients requiring close observation after surgery.

A critical complication of an anterior circulation ischaemic stroke, due to internal carotid artery (ICA) damage, for example, is malignant middle cerebral artery (MCA) syndrome. This space-occupying brain swelling occurs in a reported $2 \%$ of anterior circulation strokes with an overall incidence of 10 in 100,000 and, if untreated, carries a mortality rate of about $80 \%$ $[5,6]$. Emergency decompressive craniectomy is now the recognized treatment of choice, although outcomes remain poor in many individuals [7].

\section{Case Presentation}

A male patient in his mid-twenties was admitted to the Emergency Department (ED) having sustained a GSW to the left side of his neck. With a Glasgow Coma Scale (GCS) of 3, he was treated at the scene by the regional Helicopter Emergency Medical Service. He was intubated, ventilated, and given an emergency unit of packed red cells and tranexamic acid before being transferred to a local trauma centre.

On arrival at the ED, examination revealed a GSW to the left side of the neck underneath the angle of the jaw, with no exit wound. No other injuries were found. Initial physical neurological examination was attempted, but the low GCS proved difficult to perform. He was taken to imaging for a CT head, neck, chest, and abdomen to ascertain the extent of injuries sustained but became haemodynamically unstable (systolic blood pressure of $60 \mathrm{~mm} \mathrm{Hg}$ ) and required further units of packed red cells, as well as metaraminol. Subsequent imaging revealed leftsided vertebral artery and ICA damage, with the bullet lodged at the level C4 (shown in Fig. 1, 2). He was taken from imaging to the operating theatre for emergency exploration and ligation of damaged vessels.

Upon assessment in the theatre, the patient had a $2-\mathrm{cm}$ penetrating bullet entry site in zone III (area between the angle of the mandible and the base of the skull) of the left side of the neck, anterior to the left ear, with tracking towards the anterior triangle of the neck. He had sustained a complete transection of his left ICA, a partial transection of his left internal jugular vein, and a left parotid gland injury.

To manage the injuries sustained, the internal jugular vein was protected in a sling, and proximal control of the common carotid artery was gained using a sling and vascular clamp. Dissection was continued distally to expose the bifurcation of the common carotid artery and proximal control of the ICA. External carotid artery was achieved with a sling and clamp. The patient became more unstable (systolic blood pressure $<70 \mathrm{~mm} \mathrm{Hg}$ ), and after discussion with the second on-call trauma surgeon, the ICA was ligated using a damage control surgery (DCS) approach. The bullet, measuring $1 \mathrm{~cm}$ in diameter, was removed. Haemostasis of the bullet cavity was achieved, and the patient was sent to the intensive care unit (ICU) for further monitoring.

The patient remained intubated and ventilated for the first $24 \mathrm{~h}$ in ICU, and an initial National Institutes of Health Stroke Scale (NIHSS) was calculated to be 26. On day 2 after

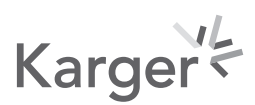




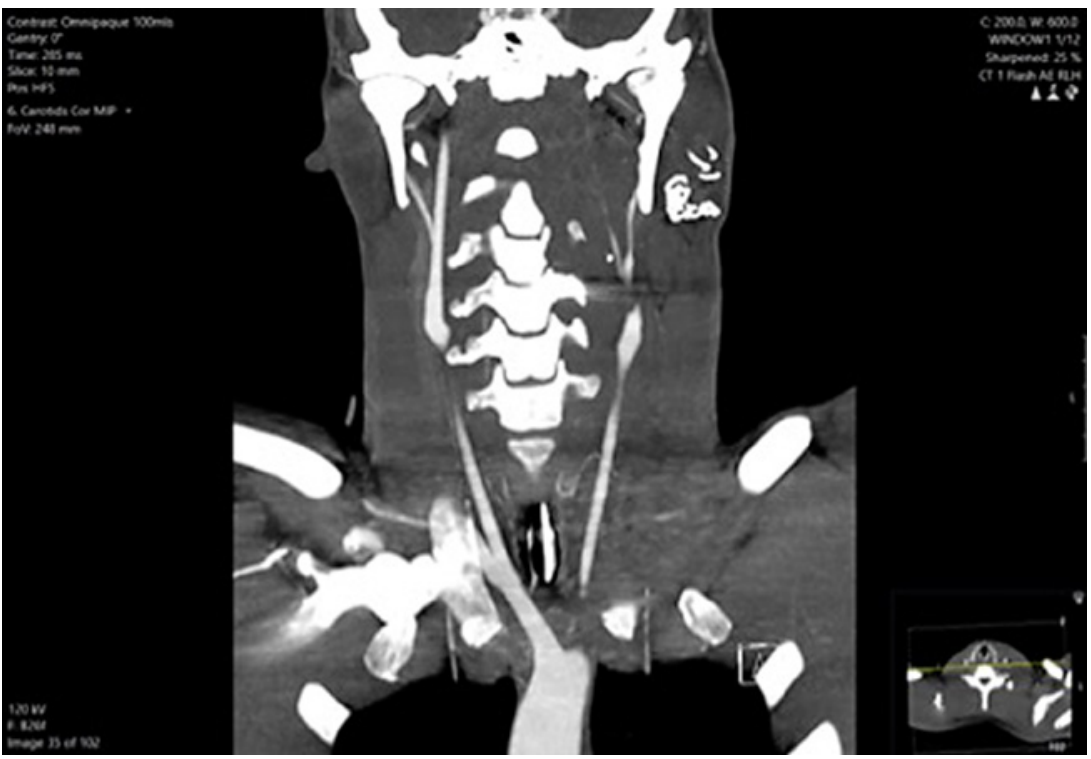

Fig. 1. Admission CT angiogram of the aortic arch and carotids: frontal/coronal views of the neck vasculature demonstrating the disruption of the left vertebral artery and subsequent distal occlusion from the level of C6/7. There is traumatic dissection and occlusion of the left ICA shortly after bifurcation. ICA, internal carotid artery.

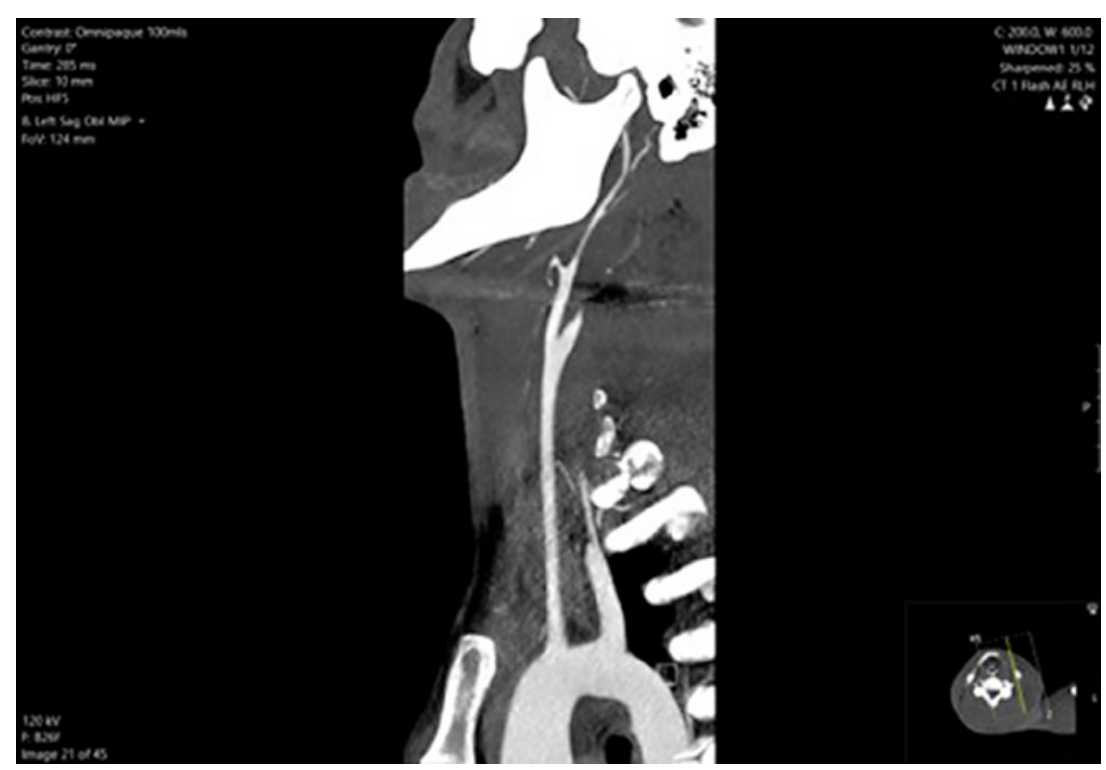

Fig. 2. Sagittal views of the neck vasculature demonstrating the abrupt occlusion of the left ICA shortly after bifurcation. ICA, internal carotid artery.

operation, examination revealed a fixed and dilated left pupil, in line with an acute neurological deterioration. An urgent CT head showed extensive ischaemic changes in the left cerebral hemisphere, particularly in the left frontal lobe. Importantly, the scan also revealed extensive parenchymal oedema with an 8-mm rightward midline shift and transtentorial herniation, in keeping with malignant MCA syndrome (shown in Fig. 3).

After timely discussions with the stroke clinicians, neurosurgeons, and family members, the patient underwent emergency surgery to reduce intracranial pressure. Three burr holes 


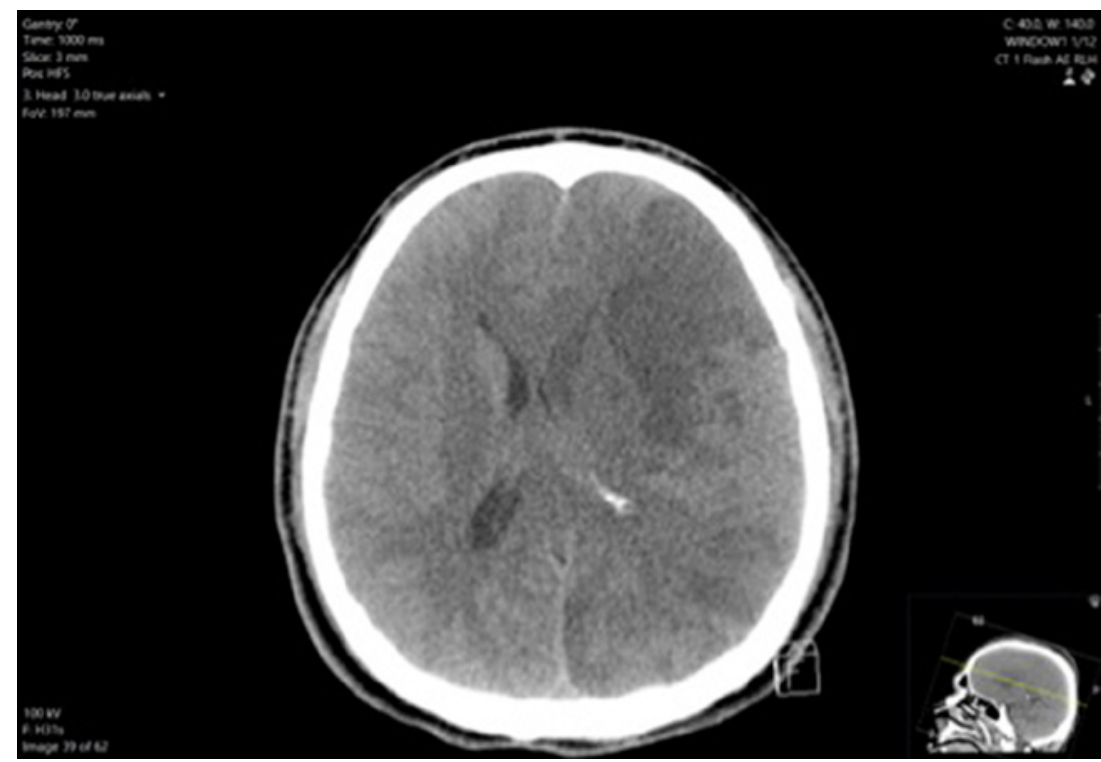

Fig. 3. CT head 2 days after admission demonstrating extensive ischaemic changes involving the left cerebral hemisphere, particularly the left frontal lobe. There is extensive cytotoxic parenchymal oedema and $8 \mathrm{~mm}$ of rightwards midline shift in keeping with malignant MCA syndrome secondary to ICA ligation. MCA, middle cerebral artery; ICA, internal carotid artery.

placed at the pterional, parasagittal anterior, and parietal-occipital regions were subsequently converted to a left decompressive temporal craniectomy with a bony window of approximately 13.5 by $14 \mathrm{~cm}$. The dura was incised and reflected, and the brain was seen to relax immediately after. Following the procedure, pupil size was noted to be 3 and $2 \mathrm{~mm}$ on the left and right, respectively (previously 6 and $2 \mathrm{~mm}$ ).

The patient was returned to ICU. Subsequent imaging 3 days later revealed a resolution of the midline shift and a reduction in the transtentorial herniation previously seen. Evolution of the left MCA and posterior cerebral artery ischaemic territory was noted. Following a successful wean from intubation and ventilation to a tracheostomy, neurological motor examination findings revealed $0 / 5$ power in both upper limbs and both lower limbs, yet sensation remained intact. The patient was only able to communicate through one-step commands using his eyes and remained globally aphasic and anarthric, yet he had no inattention. A repeated NIHSS at this stage was calculated as 23 , showing a small improvement from the time of admission.

\section{Outcome and Follow-Up}

The rest of the patient's ICU stay was largely uneventful. By day 40, power in his upper limbs had marginally improved to $1-2 / 5$ and in his lower limbs $2-3 / 5$. Proximal power was greater than distal power throughout. His global aphasia improved to moderate receptive and severe expressive dysphasia, and he began communicating with 1-2 words, showing consistent nonverbal communication. On day 49 , the patient was taken back to the theatre for a left-sided cranioplasty and on day 60 repatriated to a local stroke rehabilitation unit for ongoing therapies input.

Following repatriation, the patient remained stable and engaged consistently with physiotherapy with an improved NIHSS of 7. By day 80, power in the right lower limb had returned to normal, and the left lower limb had improved to 4-5/5. Power in both proximal upper limbs had improved to $3 / 5$, but distal power remained weaker. With regular family visitation, 
the patient's language and vocabulary improved dramatically, communicating with new words each day. He still had ongoing mild expressive dysphagia yet had more ease communicating in his native language.

On day 106, the patient successfully walked independently out of the hospital, having been discharged to a neurorehabilitation specialist centre to aid his ongoing recovery, with an NIHSS of 3. At 5 months after discharge, he continues to improve his mobility and communication skills, with new words spoken every day. His NIHSS has improved to 1 as he remains mildly dysarthric.

\section{Discussion}

This case aims to highlight the rarity of ICA transection secondary to a GSW and the subsequent malignant MCA syndrome following artery ligation. Although DCS was required due to the patient's haemodynamic instability in the theatre and the artery ligation was lifesaving, the resulting malignant MCA syndrome that followed was life threatening. Despite this, timely recognition, careful discussion between clinicians and family members, and further extensive surgery allowed the patient to begin a long but successful road to recovery, highlighted by his NIHSS improving from 26 to 1 .

\section{Carotid Artery Injury}

Penetrating carotid injuries occur in roughly $5 \%$ of penetrating neck injuries [3]. The mortality rate of untreated carotid injuries is close to $100 \%$, but in those who survive surgical intervention, the mortality rate decreases to between 6 and 33\% [8, 9]. Whilst open surgery remains the gold standard for penetrating carotid injuries, there is growing evidence for attempting an endovascular approach in more stable patients [10].

The 2 main approaches of open surgery are vessel repair and vessel ligation. Vessel repair is widely regarded as the optimal approach as it offers higher survival rates and reduced risks of neurological deficit after surgery [4]. Ligation is associated with higher rates of mortality and subsequent stroke and should be reserved for circumstances where repair is not achievable. These circumstances remain controversial among a variety of studies, although indications for ligation include systolic blood pressure $<70 \mathrm{~mm} \mathrm{Hg}$, unconscious or bilateral mydriasis, Abbreviated Injury Score (AIS) $=5$, and zone III ICA injury, in addition to established infarct on CT and neurologically intact patients [4].

In a retrospective study of penetrating injuries in patients without neurological deficits, the rate of stroke after the procedure was $8 \%$ in the vessel repair group compared with $50 \%$ in those that underwent vessel ligation, further reinforcing vessel repair as the intervention of choice [9]. However, the patient we described filled multiple criteria for undergoing vessel ligation: a penetrating injury to zone III of his neck, became unstable in the theatre with a systolic blood pressure $<70 \mathrm{~mm} \mathrm{Hg}$, and had an AIS of 5. Therefore, although ligation was the correct management in this case, the patient's risk of stroke was immediately increased. Arguably, this increased risk was unavoidable.

More recently, there has been emerging evidence surrounding endovascular techniques to treat carotid injuries, either alone or in conjunction with an open approach. A study reporting the use of an endovascular technique alone in 19 of 128 penetrating carotid injuries showed a $100 \%$ technical success rate, a $5 \%$ mortality rate, and a $5 \%$ rate of subsequent stroke [11]. As recently as September 2020, a patient with a penetrating injury to zone II of the neck was managed completely endovascularly, repairing injuries to both the arterial and venous system without the need for open surgery [12].

However, there is an absence of large studies assessing the effectiveness and outcomes of endovascular management of carotid injuries. Whilst the above examples suggest that an

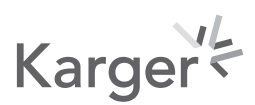


endovascular procedure may be an appropriate approach (particularly for injuries difficult to access such as the distal ICA), there is not yet enough recognized evidence to suggest an endovascular approach has better outcomes. Nor is there evidence to show this approach can be used in compromised, haemodynamically unstable patients. Whilst additional future studies may prove otherwise, we maintain that an open surgical approach in this patient was the appropriate intervention.

\section{Malignant MCA Syndrome}

Malignant MCA syndrome is an acute neurological deterioration secondary to the effects of cerebral oedema following an MCA territory infarct. There are several reasons why morbidity and mortality are high. As it is defined by an MCA territory stroke of $>50 \%$ and a perfusion deficit of $>66 \%$ on CT, the large area of the brain tissue involved creates significant cerebral oedema and in turn highly increased intracranial pressure [13]. Midline shift, secondary to oedema, compresses medial cerebral structures, and there is further potential for transtentorial herniation with compression of the posterior cerebral artery.

A recognized treatment for malignant MCA syndrome is decompressive craniectomy. It has the following effects: reduction of intracranial pressure by increasing cranial compliance, prevention of transtentorial herniation, and the improvement of perfusion of the penumbra of the stroke [7].

Although it is now the mainstay of treatment for malignant MCA syndrome, the evidence base is limited to only a handful of small trials. However, in the latest 2019 NICE guidance, it was concluded that in those under 60 years, there is a clinically important benefit of surgery compared with standard care, shown by a reduced mortality at 30 days, 6 months, and 1 year [6]. Additionally, surgery reduces patients' modified Rankin Scale (mRS), which measures the degree of disability following a stroke, to $<3$ at 6 months and 1 year $(3=$ moderate disability, requiring some help but able to mobilize independently).

Previous studies suggested that decompressive craniectomy should be reserved for $<60$ year olds, yet the updated guidance, following the DESTINY II trial [14], suggests there is also a clinically important benefit of surgery in over 60 year olds as well. Importantly though, guidance does not suggest that patients' mRS can be reduced at 6 months after surgery, meaning overall outcomes remain poor, despite a reduction in mortality rates.

Surgery warrants careful discussion between clinicians, surgeons, the patient, and the family members given the overall poor outcomes after surgery, and decisions to undergo surgery should be made on a case by case basis. In this case, it was vital the timely discussions were had due to the patient's acute deterioration, and all parties (clinicians, surgeons, and family members) were in agreement that surgery should be attempted. Ultimately, the decision was warranted, shown by the remarkable recovery made by the patient as he was eventually able to walk out of the hospital independently. At 5 months after discharge, he continues to rehabilitate with great success, and his mobility and communication skills are improving each week.

\section{Conclusion}

Penetrating GSWs are rare mechanisms of injury in the UK. In such patients, emergency imaging and rapid access to the theatre may be vital to stem the effects of such devastating injuries. DCS may be required if patients become haemodynamically unstable in the theatre. In this case, ICA ligation was necessitated and is an important cause of the resultant malignant MCA syndrome.

Open repair of extracranial vessels remains the favoured option for penetrating injuries. However, in some scenarios (poor vessel accessibility, haemodynamic instability, and high AIS),

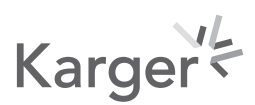


artery ligation must be considered. This carries a higher mortality and greater risk of neurological deficit after surgery. Endovascular techniques may be emerging as an alternative option but require further studies to support their routine use.

Finally, despite evidence from a relatively limited number of small studies, decompressive craniectomy remains the mainstay of treatment for malignant MCA syndrome. The outcome of many patients remains poor, yet there are examples of patients making a remarkable recovery following surgery. Timely discussions with clinicians, surgeons, and family members are very important in the decision-making process, and decisions should be made on a case by case basis.

\section{Statement of Ethics}

The authors declare that the subject in the case report has given his written informed consent to publish this case (including publication of images).

\section{Conflict of Interest Statement}

The authors have no conflicts of interest to declare.

\section{Funding Sources}

The authors declare no funding sources.

\section{Author Contributions}

G.C. and A.B. contributed equally to the concept, data collection, and manuscript writing of the case report.

\section{References}

1 Stroke statistics [Internet]. Stroke Association. 2020 [cited 2020 Nov 25]. Available from: https://www. stroke.org.uk/what-is-stroke/stroke-statistics.

2 Kowalski RG, Haarbauer-Krupa JK, Bell JM, Corrigan JD, Hammond FM, Torbey MT, et al. Acute ischemic stroke after moderate to severe traumatic brain injury: incidence and impact on outcome. Stroke. 2017 Jul;48(7):1802-9.

3 Lee TS, Ducic Y, Gordin E, Stroman D. Management of carotid artery trauma. Craniomaxillofac Trauma Reconstr. 2014 Sep;7(3):175-89.

4 Reva VA, Pronchenko AA, Samokhvalov IM. Operative management of penetrating carotid artery injuries [Internet]. Eur J Vasc Endovasc Surg. 2011 [cited 2020 Nov 25];42(1):16-20.

5 Heiss W-D. Malignant MCA infarction: pathophysiology and imaging for early diagnosis and management decisions. Cerebrovasc Dis. 2016;41(1-2):1-7.

6 Recommendations|Stroke and transient ischaemic attack in over 16s: diagnosis and initial management|Guidance|NICE [Internet]. NICE; [cited 2020 Dec 31]. Available from: https://www.nice.org.uk/ guidance/ng128/chapter/Recommendations\#decompressive-hemicraniectomy.

7 Yang MH, Lin H-Y, Fu J, Roodrajeetsing G, Shi S-L, Xiao S-W. Decompressive hemicraniectomy in patients with malignant middle cerebral artery infarction: a systematic review and meta-analysis. Surgeon. 2015 [cited 2020 Nov 19];13(4):230-40.

8 Navsaria P, Omoshoro-Jones J, Nicol A. An analysis of 32 surgically managed penetrating carotid artery injuries [Internet]. Eur J Vasc Endovasc Surg. 2002 [cited 2020 Nov 25];24(4):349-55.

9 Ramadan F, Rutledge R, Oller D, Howell P, Baker C, Keagy B. Carotid artery trauma: a review of contemporary trauma center experiences [Internet]. J Vasc Surg. 1995 [cited 2020 Nov 25];21(1):46-55. 
10 DuBose J, Recinos G, Teixeira PGR, Inaba K, Demetriades D. Endovascular stenting for the treatment of traumatic internal carotid injuries: expanding experience. J Trauma. 2008 Dec;65(6):1561-6.

11 du Toit DF, Coolen D, Lambrechts A, de V Odendaal J, Warren BL. The endovascular management of penetrating carotid artery injuries: long-term follow-up [Internet]. Eur J Vasc Endovasc Surg. 2009 [cited 2020 Nov 25];38(3):267-72.

12 Kaslow SR, Gurney 0, Ascher E. Explore no more: early experience with a novel minimally invasive approach to penetrating trauma to zone II of the neck. J Vasc Surg Cases Innov Tech. 2020 Sep 8;6(4):595-7.

13 Treadwell SD, Thanvi B. Malignant middle cerebral artery (MCA) infarction: pathophysiology, diagnosis and management. Postgrad Med J. 2010 Apr 1;86(1014):235-42.

14 Jüttler E, Bösel J, Amiri H, Schiller P, Limprecht R, Hacke W, et al. DESTINY II: DEcompressive surgery for the treatment of malignant INfarction of the middle cerebral arterY II. Int J Stroke. 2011 Feb;6(1):79-86. 